Related content

\title{
A hybrid light source with integrated inorganic light-emitting diode and organic polymer distributed feedback grating
}

To cite this article: Bayram Butun et al 2008 Nanotechnology 19195202

\author{
Optical gain characteristics of beta-phase \\ poly(9.9-dioctylfluorene) \\ G Ryu, R Xia and D D C Bradley \\ - INVITED PAPER \\ C R Belton, G Itskos, G Heliotis et al. \\ Enhancement of the mode coupling in \\ photonic-crystal-based orqanic lasers \\ Rik Harbers, Nikolaj Moll, Rainer F Mahrt \\ et al.
}

\section{Recent citations}

View the article online for updates and enhancements.

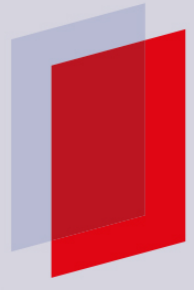

\section{IOP ebooks'}

Bringing you innovative digital publishing with leading voices to create your essential collection of books in STEM research. Start exploring the collection - download the first chapter of every title for free. 


\title{
A hybrid light source with integrated inorganic light-emitting diode and organic polymer distributed feedback grating
}

\author{
Bayram Butun ${ }^{1,4,5}$, Koray Aydin ${ }^{1,5}$, Erkin Ulker $^{1}$, \\ Stéphanie Cheylan ${ }^{2}$, Gonçal Badenes ${ }^{2}$, Michael Forster ${ }^{3}$, \\ Ullrich Scherf $^{3}$ and Ekmel Ozbay ${ }^{1}$ \\ ${ }^{1}$ Nanotechnology Research Center, Department of Physics, Department of Electrical and
Electronics Engineering, Bilkent University, Bilkent, 06800 Ankara, Turkey
${ }^{2}$ ICFO_Institut de Ciències Fotòniques, Mediterranean Technology Park, Avenida Canal
Olímpic s/n, E-08860 Castelldefels (Barcelona), Spain
${ }^{3}$ Bergische Universität Wuppertal, Makromolekulare Chemie, Fachbereich Chemie,
D-42097 Wuppertal, Germany \\ E-mail: bbtn@ee.bilkent.edu.tr
}

Received 25 February 2008, in final form 7 March 2008

Published 7 April 2008

Online at stacks.iop.org/Nano/19/195202

\begin{abstract}
We report a compact light source that incorporates a semiconductor light-emitting diode, nanostructured distributed feedback (DFB) Bragg grating and spin-coated thin conjugated polymer film. With this hybrid structure, we transferred electrically generated $390 \mathrm{~nm}$ ultraviolet light to an organic polymer via optical pumping and out-couple green luminescence to air through a second-order DFB grating. We demonstrate the feasibility of electrically driven, hybrid, compact light-emitting devices and lasers in the visible range.
\end{abstract}

(Some figures in this article are in colour only in the electronic version)

In recent years, conjugated polymers have attracted much interest due to their high luminescence quantum yield [1], easy synthesis, broad and chemically adjustable emission wavelength range in the visible spectrum [2], minimized selfabsorption due to very weak sub-bandgap absorptions [3], and low cost [4]. Near-zero self-absorption above the absorption edge and rather low energy thresholds for amplified spontaneous emission (ASE) makes them rather attractive for wavelength conversion with high throughput and novel laser devices. It is also possible to dope them with other chromophores [5] for multimode operation or to obtain tunable organic lasers via continuous modification of the grating used in the devices [6]. White light generation using a commercial light-emitting diode (LED) as a pump and a polymer as the white light luminescent layer [7-9] was also reported.

Optically pumped laser action in conjugated polymers and copolymers has been demonstrated with an emission across the whole visible spectrum (blue, green and red) and

4 Author to whom any correspondence should be addressed.

5 These authors contributed equally to this work. for different resonator geometries (microcavity, distributed feedback (DFB), distributed Bragg reflector (DBR), etc) by several research groups [10-15]. Although high efficiency organic LEDs are now achievable, the development of electrically pumped organic lasers remains an outstanding, and up to now unsolved, challenge. One fundamental reason for the failure is, first, the high current density needed to realize an electrically pumped, amplified spontaneous emission, and second, the presence of metal contacts (electrodes) that cause a big portion of optical loss. Finally, the charged states of the organic materials (polarons, bipolarons) which are generated during the charge carrier injection at the electrodes also absorb light, and therefore increase the energy threshold for amplified spontaneous emission. The realization of an organic materialbased diode laser requires a complex optimization of optical and electrical properties of all components as well as of the device geometry. Regarding inorganic materials, epitaxially grown GaN-based inorganic semiconductor structures have found their application as efficient, robust, visible and ultraviolet (UV) light sources. GaN structures offer excellent 

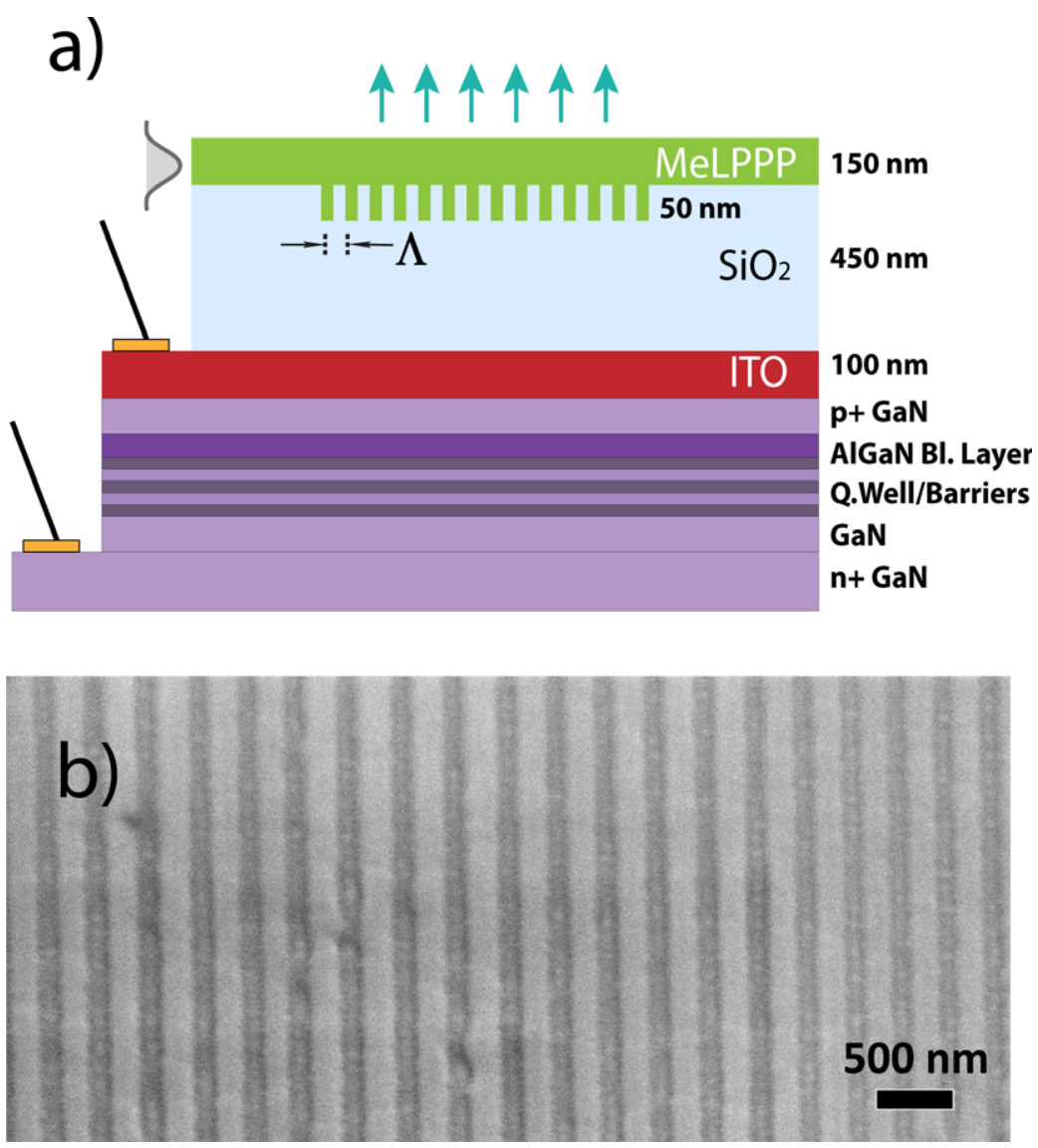

Figure 1. (a) Schematic diagram of the fabricated LED structure and $\mathrm{SiO}_{2}$ DFB grating with MeLPPP layer. (b) SEM image of the patterned area after FIB milling process with a grating period of $310 \mathrm{~nm}$.

electrical properties [16] but their spectral coverage is limited. However, down-conversion with phosphors enables access to other colors and white light emission. Although blueemitting III-V semiconductor laser diodes ( $\mathrm{GaN}$, InGaN) have recently been proposed as a pump source for organic materials [9, 17-19], it is noteworthy that there have been no reports to date of a compact, integrated device such as the one proposed in the present paper that may pave the way towards color-tunable LEDs and inorganic/organic hybrid lasers. Hybrid light-emitting devices incorporating GaN light sources with organic polymers were previously reported [7, 9]. Hide et al used lenses to focus light emitted from a GaNLED [7]. Heliotis et al stacked GaN-LED and an organic polymer coated on top of a quartz substrate [9]. Our structure is a compact light-emitting device where organic polymer is coated on top of a GaN-LED and includes a filtering feedback mechanism based on distributed gratings.

Taking into account that lasing thresholds of polymer DFB lasers are now commonly low enough to be pumped by pulsed microchip lasers [20], we tested the combination of a GaNbased LED as the light source and polymeric laser materials embedded in a suitable grating structure in order to develop a compact light-emitting device structure with a future potential for electrically pumped organic lasing. In the present work, we first grew and fabricated a UV-visible InGaN/GaN-LED. We then patterned nanometer-sized DFB gratings on top of the device by a focused ion beam (FIB) milling technique, and coated the entire surface with the conjugated para-phenylenetype ladder polymer MeLPPP that is known for its low ASE threshold (a maximum ASE cross section of $\sim 1.5 \times 10^{-16} \mathrm{~cm}^{2}$ was observed at a wavelength of $490 \mathrm{~nm}$ ). In this device configuration, the polymer was pumped by a GaN-based LED and the second-order Bragg grating selectively coupled the down-converted photons to air (figure 1(a)).

The emitter polymer that was used in our study was a para-phenylene ladder polymer MeLPPP [1], which is a fully planarized, rigid conjugated polymer without any considerable conformational distortion. Together with its high fluorescence quantum yield in the solid state (approx. 40-50\%) and the fully amorphous solid state structure, it is currently one of the conjugated polymers with the highest gain value for stimulated emission. MeLPPP is an ideal candidate for basic energy transfer studies in inorganic-organic hybrid devices [2]. The absorption band is well resolved without significant inhomogeneous broadening with a (0-0) absorption maximum centered around $450 \mathrm{~nm}$. The fluorescence emission peak (00) shows a very small Stokes shift (approx. $150 \mathrm{~cm}^{-1}$ ) due to the very rigid ladder structure of the conjugated backbone. Due to the lack of self-absorption in the spectral region $>470 \mathrm{~nm}$ the $(0-1)$ emission band shows a much higher gain when compared to the higher energy (0-0) transition. MeLPPP has been extensively studied for its photo- and 

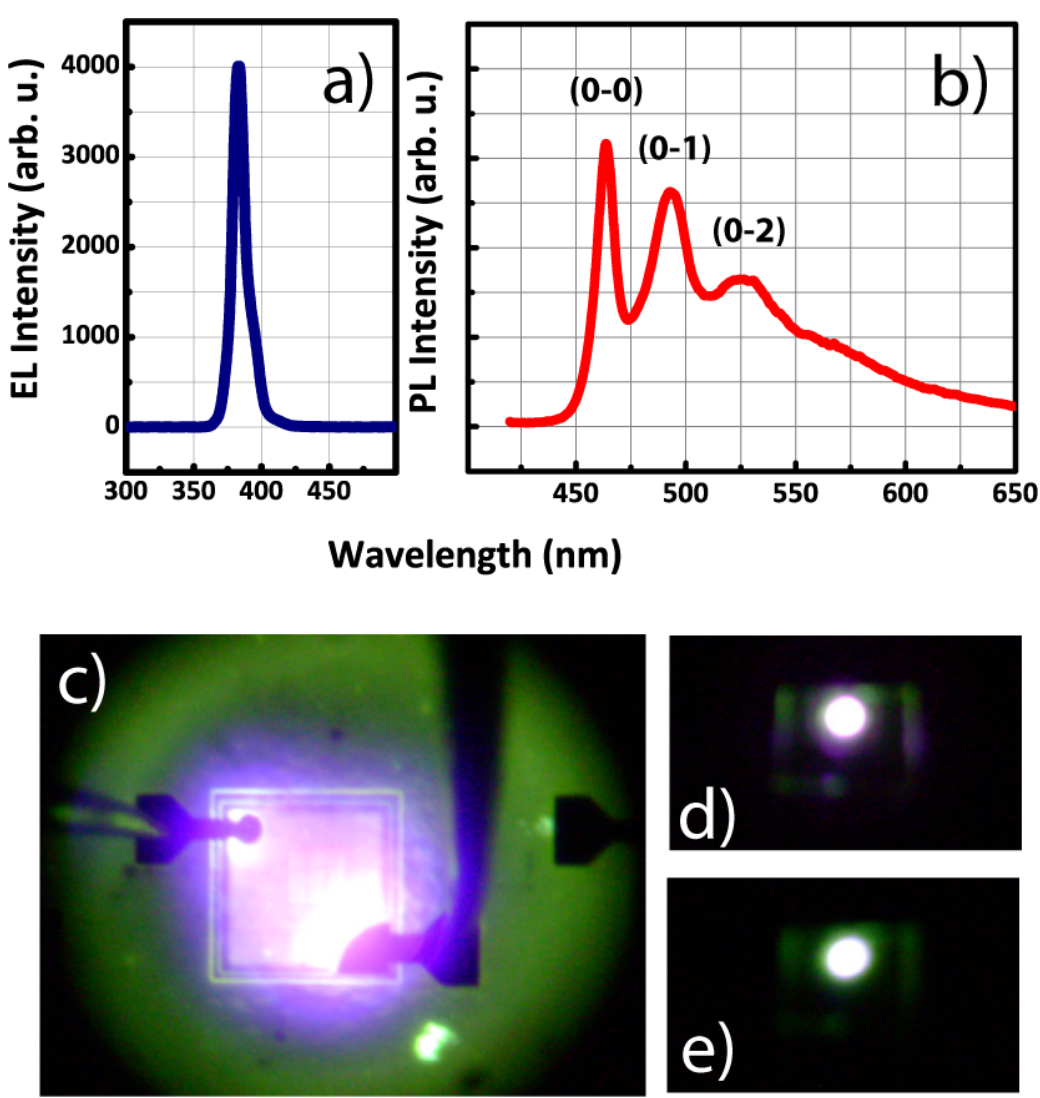

Figure 2. (a) Electroluminescence spectrum of the fabricated GaN-LED. (b) Photoluminescence spectrum of the organic MELPPP layer on top of a sapphire substrate. (c) Photograph of the hybrid LED device in electroluminescence; (d) far-field image and (e) far-field image with a $400 \mathrm{~nm}$ cutoff high-pass filter.

electroluminescence properties including its ASE and lasing behavior in optically pumped solid state polymer lasers with DFB and DBR resonator geometry $[2,15,21]$.

The LED wafer was grown on a $c$-plane (0001) sapphire substrate by the AIXTRON RF200/4 metal-organic chemical vapor deposition (MOCVD) system at the Bilkent University Nanotechnology Research Center [22]. First, a GaN nucleation layer was grown followed by a $500 \mathrm{~nm}$ thick $\mathrm{GaN}$ buffer layer. Then, an $\mathrm{n}+\mathrm{GaN}$ layer was grown and 5 pairs of $\mathrm{InGaN} / \mathrm{GaN}$ were grown with a total thickness of 80-100 nm. To increase the charge recombination and enhance the light output, a $20 \mathrm{~nm}$ thick p-doped AlGaN layer was grown before the p-doped $\mathrm{GaN}$ top layer. Diodes are fabricated in a class-100 cleanroom environment with standard micro-fabrication techniques [23]. Since GaN-based structures are best processed via reactive ion etching (RIE) instead of wet etching techniques, all of the etching steps were performed before any metallization. First, the wafer was etched down to the $n+$ layer, and with a mesa mask. Later on, it was etched down to the GaN buffer. Thereafter, 10/100 nm thick Ti/Au metals for $\mathrm{n}+$ ohmic contact and $75 \mathrm{~nm}$ thick indium tin oxide (ITO) for $\mathrm{p}+$ ohmic contact metals were deposited by evaporation and subsequently lifted off. The contacts were annealed at $650^{\circ} \mathrm{C}$ for $2 \mathrm{~min}$. The touch pad to $\mathrm{p}+$ contact was $10 / 100 \mathrm{~nm} \mathrm{Ni} / \mathrm{Au}$ and the contact pads for the measurements were thick Ti/Au metals. Before the contact pad, the diode surfaces were coated with insulating dielectric films of $\mathrm{SiO}_{2}$ using plasma-enhanced chemical vapor deposition (PECVD) and etched by an $\mathrm{HF} / \mathrm{H}_{2} \mathrm{O}$ solution. The LEDs on the sample have areas ranging from $160 \times 160$ to $480 \times 480 \mu \mathrm{m}^{2}$. Fabricated LEDs have turn-on voltages of approx. $5 \mathrm{~V}$ with $1 \mathrm{~mA}$ forward current. At 6 and $7 \mathrm{~V}$, the current levels were 9 and $29 \mathrm{~mA}$, respectively. Figure 2(a) displays the electroluminescence (EL) spectrum of the GaNLED device under test. The emission peak appears at $390 \mathrm{~nm}$.

The fabrication of the gratings on the GaN-LED's top electrode was performed at ICFO by using an FIB system (FEI Strata DB235). A very thin layer of gold (Au) was evaporated on top of the devices in order to prevent charging effects. We fabricated one-dimensional linear gratings of an area approx. $100 \times 100 \mu \mathrm{m}^{2}$ by FIB milling, partially covering the LED surface (figure 1(b)). The gratings had $50 \mathrm{~nm}$ deep trenches with periods of $\Lambda=300$ and $310 \mathrm{~nm}$. The milling time was approx. 2-3 h long for a single patterning of $100 \mu \mathrm{m} \times 100 \mu \mathrm{m}$, using currents of the order of $100 \mathrm{pA}$.

The organic polymer was dissolved in toluene with a concentration of $\sim 25 \mathrm{mg} \mathrm{ml}^{-1}$ with the help of an ultrasound bath and spin-coated on the surface of the LED device at $2500 \mathrm{rpm}$ (for $30 \mathrm{~s}$ ) resulting in a $150 \mathrm{~nm}$ thick organic layer. The thickness of the organic layer was selected to be $>\lambda / 2 n$ of the emission wavelength of interest (about $490 \mathrm{~nm}$ ) to obtain an asymmetric waveguide, supporting only the first mode. The photoluminescence spectrum of the MeLPPP layer on top of the sapphire substrate is shown in figure 2(b). Three 


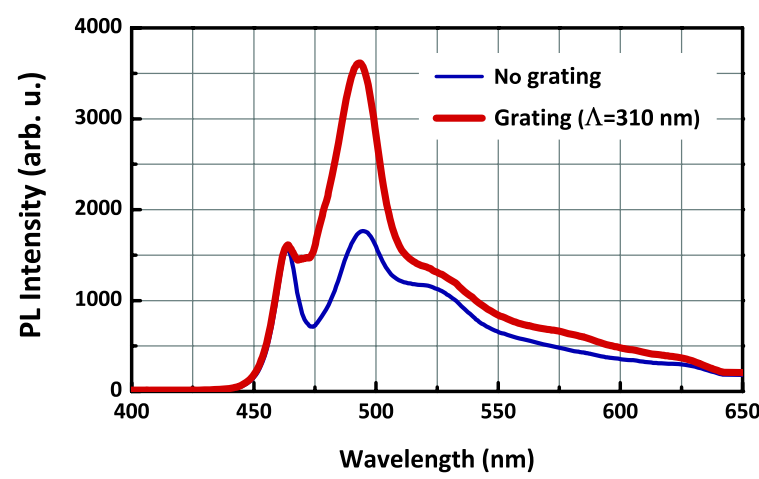

Figure 3. PL of the hybrid LED device with a grating period of $310 \mathrm{~nm}$ using a fiber probe for light collection.

distinct emission peaks are present at wavelengths 460, 490 and $525 \mathrm{~nm}$.

Figure 1 shows the schematic structure of the complete device. The MeLPPP polymer $(n=1.75)$ layer was sandwiched between air and a $450 \mathrm{~nm} \mathrm{SiO}{ }_{2}(n=1.46)$ layer in an asymmetric waveguide structure. In comparison to $\mathrm{SiO}_{2}$, ITO shows a much higher refractive index of 2.05 . The refractive index of $\mathrm{GaN}$ is $\sim 2.35$. Therefore, the $\mathrm{SiO}_{2}$ layer should be as thick as possible in order to prevent evanescent wave losses. Emitted light corresponding to a Bragg wavelength of $490 \mathrm{~nm}$ will be decoupled to air through the distributed feedback (DFB) grating. An SEM image of the $\mathrm{SiO}_{2}$-based DFB grating is shown in figure 1(b) demonstrating a highly uniform grating structure, which is rather important for the high light output of the device.

In figure 2(c), a CCD image of an LED device is shown under $6 \mathrm{~V}$ bias and $9 \mathrm{~mA}$ current. The white areas on the device mesa show that the combination of blue LED emission and optically pumped MeLPPP luminescence effectively produces a broad spectrum, mostly covering the blue and green components of visible light. The grating region in the middle of the device is not clearly visible since the image is mainly dominated by the LED emission. The farfield image of the same sample without a filter is shown in figure 2(d). Here, the GaN-LED emission is also apparent from the edges of the device due to the total internal reflection. A similar image through a high-pass filter with cutoff at $400 \mathrm{~nm}$ is shown in figure 2(e). The $390 \mathrm{~nm}$ emission of the LED is now blocked with the filter. The emitted light now originates from the organic layer, indicating a uniform absorption of the LED emission by the organic layer. We performed transmission and reflection measurements of $\mathrm{MeLPPP} / \mathrm{SiO}_{2}(150 \mathrm{~nm} / 450 \mathrm{~nm})$ coated sapphire samples using an Ocean Optics spectrometer around $400 \mathrm{~nm}$. We obtained the absorption of polymer film approximately $12 \%$. Since ideally a complete absorption by the polymer would be preferred, either a thicker MeLPPP layer or a pump LED with an emission near the absorption maximum of MeLPPP ( $450 \mathrm{~nm}$ ) should be utilized.

To characterize the hybrid devices, we performed electroluminescence (EL) and photoluminescence (PL) measurements on the patterned and non-patterned areas and with/without spin-coated organic material. Emission from the grating was collected with a $125 \mu \mathrm{m}$ diameter multimode fiber

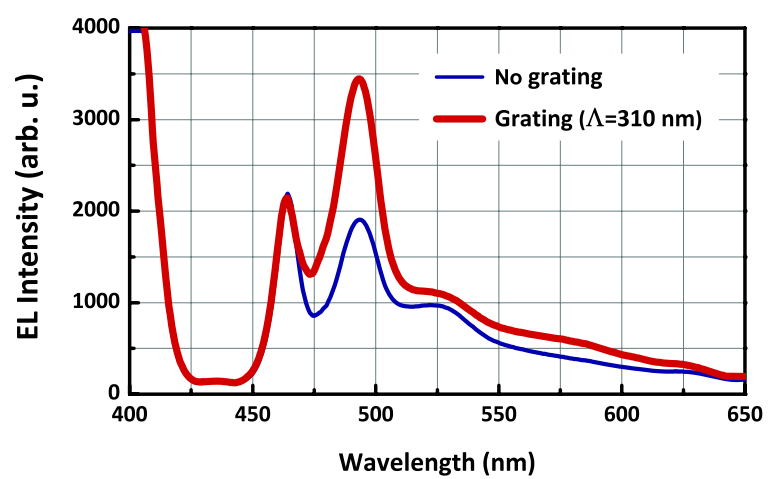

Figure 4. EL of the hybrid device with a grating period of $310 \mathrm{~nm}$.

probe connected to a spectrometer in the wavelength range of $400-650 \mathrm{~nm}$.

We first performed photoluminescence measurements on the hybrid device with a grating period of $310 \mathrm{~nm}$. We optically pumped the MeLPPP layer close to the grating with a $325 \mathrm{~nm}$ continuous wave (CW) HeCd laser and collected the decoupled light with the fiber probe. Figure 3 shows the PL spectra of the hybrid LED device without grating (blue line) and with $310 \mathrm{~nm}$ periodicity grating (red line). We observed a 2.06fold increase in the intensity of the (0-1) MeLPPP emission band (490 nm emission peak) within the grating region. The Bragg condition is given as $\Lambda=m \lambda / 2 n_{\text {eff }}$, where $\Lambda$ is the grating period, $m$ is the order of the grating and $\lambda$ is the Bragg wavelength. $n_{\text {eff }}$ denotes the effective refractive index of the supported waveguide mode. In this work, we utilized a second-order Bragg grating to have a vertical emission of light. In order to match the second-order Bragg condition with the equation [4] $\Lambda=\lambda / n_{\text {eff }}$, the grating period should be around $\Lambda=314 \mathrm{~nm}$. We calculated $n_{\text {eff }}$ to be around 1.57 using the device parameters, thicknesses of the organic and dielectric layers and grating depth and periods [24]. For a grating with a periodicity of $310 \mathrm{~nm}$, the Bragg wavelength corresponds to $487 \mathrm{~nm}$, which clearly is within the (0-1) emission band.

We then performed electroluminescence measurements. We applied voltage to the LED device and measured light outgoing from the organic surface. Here, the main idea is to use the inorganic light-emitting device to convert its emission energy down to higher wavelengths, namely the wavelength of the (0-1) emission band of the conjugated polymer MeLPPP. The EL spectra of devices without (blue line) and with a grating period of $310 \mathrm{~nm}$ (red line) are given in figure 4 . Different from the PL spectra, we observed another emission wavelength that is around $400 \mathrm{~nm}$. This corresponds to the emission of the GaN-LED structure as plotted in figure 2(a). It is apparent that a part of the GaN UV emission is converted down to the lower energy MeLPPP emission region. The LED structure has an emission peak at $390 \mathrm{~nm}$ and MeLPPP absorbs the light at $390 \mathrm{~nm}$ and emits at higher wavelengths. LED emission was measured to be considerably higher than the MeLPPP emission due to low absorption of the thin polymer film (\%12). LED emission could be suppressed by using thicker organic polymer films. This result shows the feasibility of incorporating inorganic light-emitting sources with organic 


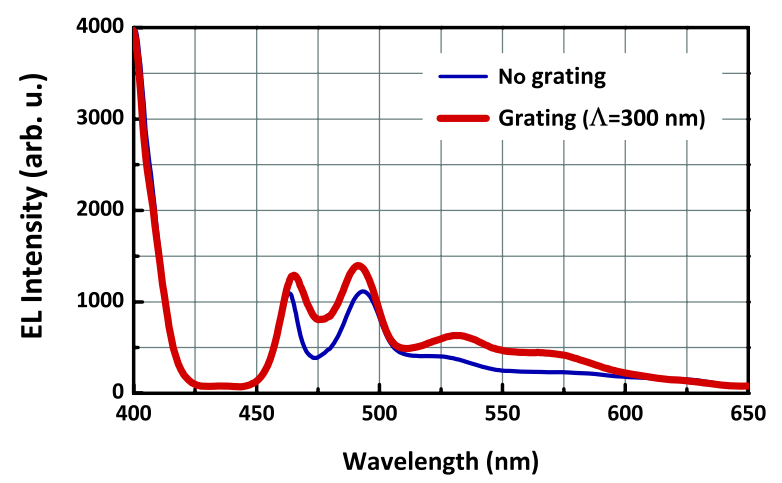

Figure 5. EL of the hybrid device with a grating period of $300 \mathrm{~nm}$.

materials in order to produce hybrid light-emitting devices. It is worthy of note that the device does not yield lasing in the polymer. The long wavelength emission bands in MeLPPP, especially in electroluminescence, have been related to the formation of keto defect states in photo- or electrooxidatively degraded MeLPPP layers that act as charge and exciton traps [25].

The electroluminescence enhancement in the region of the MeLPPP emission for a grating period of $310 \mathrm{~nm}$ and a surface depth of $50 \mathrm{~nm}$ is illustrated in figure 4 . The enhancement factor at $490 \mathrm{~nm}$ is measured to be 1.85 . We also observed EL enhancement at longer wavelengths $(>520 \mathrm{~nm})$, but the amount of enhancement is not significant compared to the wavelengths of interest (around $490 \mathrm{~nm}$ ). The fluorescence component at higher wavelength is caused by energy transfer (Foerster transfer) of the initial excitation to emissive keto defects. Enhancement in the emission at longer wavelengths may be related to the coupling of both processes (fluorescence enhancement and energy transfer). The enhancement factor could further be increased by matching the Bragg wavelength more exactly to the (0-1) emission peak of MeLPPP (490 nm). One could also fabricate deeper gratings in order to increase the amount of Bragg reflections taking place within the grating region.

We also fabricated another device with a different periodicity of Bragg grating, $300 \mathrm{~nm}$. In figure 5, EL emission spectra from the grating and flat area (area on the diode mesa but outside the grating) were recorded for a grating period of $300 \mathrm{~nm}$. For the emission at $490 \mathrm{~nm}$ wavelength the enhancement factor in the grating region was 1.27; amplification was also observed for higher wavelengths $>500 \mathrm{~nm}$. This measurement shows that the increased deviation of the grating period from the preferred value $(\sim 314 \mathrm{~nm})$ causes a decrease in the enhancement factor, as expected. The Bragg wavelength for a grating period of $300 \mathrm{~nm}$ is calculated to be $471 \mathrm{~nm}$ in the local minimum between the (0-0) and (0-1) emission features of MeLPPP. At shorter wavelengths outside the polymer emission feature the intensities of the emission bands with and without grating match perfectly, indicating that the EL enhancement only exclusively originates from the DFB grating.

In conclusion, we fabricated a high performance InGaN/GaN-based LED with $390 \mathrm{~nm}$ emission and combined it with an emissive conjugated polymer (MeLPPP) nanostructured grating for wavelength down-conversion with high wavelength selectivity and out-coupling efficiency. The grating with $310 \mathrm{~nm}$ periodicity showed a nearly twofold amplification factor for an emission wavelength of $490 \mathrm{~nm}$ corresponding to the $(0-1)$ emission band of the polymer. We showed the feasibility of a compact hybrid light-emitting device, which is electrically operated and may be easily modified for tunable wavelength emission, white light generation and multimode operation. The structure combines the superior characteristics of electrical (LED) and optical (conjugated polymer MeLPPP) components for, most importantly, future compact laser sources for the UVvisible range based on DFB or DBR resonator structures.

\section{Acknowledgments}

This work is supported by the European Union under the projects EU-NoE-METAMORPHOSE, EU-NoEPHOREMOST, EU-PHOME, EU-ECONAM, TUBITAK (under project nos 105E066, 105A005, 106E198, 106A017 and 107A012) and MEC (Consolider-Ingenio CSD2007-00007, TEC2006-10665). SC acknowledges support from the Spanish Ministry of Education and Science (Ramon y Cajal program). EO also acknowledges partial support from the Turkish Academy of Sciences.

\section{References}

[1] Schweitzer B, Wegmann G, Giessen H, Hertel D, Bassler H, Mahrt R F, Scherf U and Mullen K 1998 Appl. Phys. Lett. 722933

[2] Scherf U 1999 J. Mater. Chem. 91853

[3] Hide F, Díaz-García M A, Schwartz B J, Andersson M R, Pei Q and Heeger A J 1996 Science 2731833

[4] Bauer C, Giessen H, Schnabel B, Kley E B, Schmitt C, Scherf U and Mahrt R F 2001 Adv. Mater. 131161

[5] Sobel F, Gindre D, Nunzi J-M, Denis C, Dumarcher V, Fiorini-Debuisschert C, Kretsch K P and Rocha L 2004 Opt. Mater. 27199

[6] Wang J, Weimann T, Hinze P, Ade G, Schneider D, Rabe T, Riedl T and Kowalsky W 2005 Microelectron. Eng. 78/79 364

[7] Hide F, Kozody P, DenBaars S P and Heeger A J 1997 Appl. Phys. Lett. 702664

[8] Hide F, DenBaars S P and Heeger A J 1999 US Patent Specification 5,966,393

[9] Heliotis G, Stavrinou P N, Bradley D D C, Gu E, Griffin C, Jeon C W and Dawson M D 2005 Appl. Phys. Lett. 87103505

[10] Tessler N, Denton G J and Friend R H 1996 Nature 382695

[11] Kallinger C et al 1998 Adv. Mater. 10920

[12] Bauer C, Giessen H, Schnabel B, Kley E-B, Schmitt C, Scherf U and Mahrt R 2003 Adv. Mater. 131161

[13] Stehr J et al 2003 Adv. Mater. 151726

[14] Rothe C, Galbrecht F, Scherf U and Monkman A 2006 Adv. Mater. 182137

[15] Scherf U, Riechel S, Lemmer U and Mahrt R 2001 Curr. Opin. Solid State Mater. Sci. 5143

[16] Nakamura S 1999 J. Cryst. Growth 202290

[17] Vasdekis A E, Tsiminis G, Ribierre J-C, O' Faolain L, Krauss T F, Turnbull G A and Samuel I D W 2006 Opt. Express 149211

[18] Karnutsch C et al 2006 Appl. Phys. Lett. 89201108 
[19] Riedl T, Rabe T, Johannes H-H, Kowalsky W, Wang J, Weimann T, Hinze P, Nehls W, Farrell T and Scherf U 2006 Appl. Phys. Lett. 88241116

[20] Turnbull G A, Andrew P, Barnes W L and Samuel I D W 2003 Appl. Phys. Lett. 82313

[21] Lanzani G, De Silvestri S, Cerullo G, Stagira S, Nisoli M, Graupner W, Leising G, Scherf U and Müllen K 1999 Semiconducting Polymers ed G Hadziioannou and P F van Hutten (Heidelberg: Wiley-VCH) p 235
[22] Yu H B, Ozturk M K, Ozcelik S and Ozbay E 2006 J. Cryst. Growth 293273

[23] Butun B, Cesario J, Enoch S, Quidant R and Ozbay E 2007 Photon. Nanostruct. Fundam. Appl. 586

[24] Pollock C R 1995 Fundamentals of Optoelectronics (Chicago, IL: Richard D Irwin)

[25] Romaner L, Heimel G, Wiesenhofer H, Scandiucci de Freitas P, Scherf U, Bredas J-L, Zojer E and List E J W 2004 Chem. Mater. 164667 